\title{
Effect of collagen vascular disease-associated interstitial lung disease on the outcomes of lung cancer surgery
}

\author{
Hideyuki Maeda ${ }^{1} \cdot$ Masato Kanzaki $^{1} \cdot$ Kei Sakamoto $^{1} \cdot$ Tamami Isaka $^{1} \cdot$ \\ Kunihiro Oyama $^{1} \cdot$ Masahide Murasugi $^{1} \cdot$ Takamasa Onuki $^{1}$
}

Received: 13 September 2016 / Accepted: 4 January 2017 / Published online: 28 February 2017

(C) The Author(s) 2017. This article is published with open access at Springerlink.com

\begin{abstract}
Purpose This study compared the effect of collagen vascular disease-associated interstitial lung disease (CVDILD) with that of idiopathic interstitial pneumonias (IIPs) on the outcomes of lung cancer surgery.

Methods This study retrospectively reviewed the medical records of patients who underwent surgery for nonsmall cell lung cancer (NSCLC) and compared the data of 16 patients with CVD-ILD with those of 70 patients with IIPs. The patterns of interstitial lung disease (ILD) on chest computed tomography were classified into usual interstitial pneumonia (UIP) and non-specific interstitial pneumonia (NSIP) patterns.

Results The numbers of UIP and NSIP patterns were 10 (62.5\%) and 6 (37.5\%) patients in CVD-ILD group, and 62 (88.6\%) and $8(11.4 \%)$ patients in IIPs group, respectively. A postoperative acute exacerbation (AE) appeared in 1 patient $(6.3 \%)$ in the CVD-ILD group and 6 patients $(8.6 \%)$ in the IIPs group. No significant differences in the incidence of postoperative $\mathrm{AE}$ and mortalities were observed between the two groups. The five-year overall survival rates of the CVD-ILD and IIPs groups were 37.5 and $49.2 \%$, respectively.

Conclusions Surgery for NSCLC in CVD-ILD patients appear to cause no increase in postoperative $\mathrm{AE}$ and mortality in comparison to that seen in IIPs patients. Similar to IIPs, CVD-ILD might therefore affect the prognosis of resected NSCLC.
\end{abstract}

Masato Kanzaki

kanzaki@twmu.ac.jp

1 Department of Surgery I, Tokyo Women's Medical University, 8-1 Kawada-cho, Shinjuku-ku, Tokyo 162-8666, Japan
Keywords Lung cancer $\cdot$ Surgery $\cdot$ Interstitial lung disease $\cdot$ Collagen vascular disease

\section{Introduction}

Interstitial lung disease (ILD) frequently coexists with collagen vascular disease (CVD) in patients, and most such patients are treated with immunosuppressive agents. Lung cancer in patients with ILD has a poor prognosis, and the postoperative acute exacerbation (AE) of ILD is one of the most critical complications observed after lung resection. Recently, Fukui et al. report that patients with combined pulmonary fibrosis and emphysema show poor postoperative outcomes after lung cancer surgery [1]. Although the prognosis of CVD-associated ILD (CVD-ILD) is better than that of idiopathic interstitial pneumonias (IIPs) [2], the effect of CVD-ILD on the prognosis of lung cancer compared to that of IIPs and the incidence of postoperative AE of CVD-ILD are unknown. In addition, the use of immunosuppressive agents may increase the risk of postoperative complications, such as bronchopleural fistula (BPF). Consequently, CVD-ILD patients undergoing lung cancer surgery are speculated to have a higher risk of postoperative morbidities and mortality. However, to date, there is no report regarding the outcomes of lung cancer surgery in CVD-ILD patients. This study compared the effect of CVD-ILD with that of patients with IIPs on the outcomes of lung cancer surgery.

\section{Patients and methods}

This was a single-institutional retrospective study based on medical records obtained from the authors' institution. 
The authors reviewed the clinical data of 1114 patients who underwent lung resection for non-small cell lung cancer (NSCLC) at our institution from January 2003 to December 2014. These patients were classified into two groups, including patients with CVD $(n=40)$ and those without $(n=1074)$. Then, based on the chest computed tomography (CT) findings described below, the patients belonging to the CVD-ILD group $(n=16)$ and those belonging to the IIPs group $(n=70)$ were newly extracted from the two groups individually (Fig. 1). The postoperative follow-up period was defined as the period from operation to the last follow-up appointment.

\section{Diagnosis of interstitial lung disease}

The diagnosis of ILD was based on the following criteria, and the patterns of ILD on chest CT was classified into usual interstitial pneumonia (UIP) and non-specific interstitial pneumonia (NSIP) patterns. The criteria for the UIP pattern were (1) subpleural basal predominance, (2) reticular abnormality, (3) honeycombing with or without traction bronchiectasis, and (4) the absence of features listed as inconsistent with UIP pattern as described in the literature [3]. Any patients with a possible UIP pattern on CT [3] were excluded from this study. The criteria for the NSIP pattern were reticular opacities with a lower lung zone predominance, associated with traction bronchiectasis, and lobar volume loss [4]. CT scanned images were read by both experienced radiologists and thoracic surgeons. After surgery, all resected specimens were investigated by pathologists to confirm the existence of ILD.

\section{Prophylaxis for an acute exacerbation of interstitial pneumonia}

At the authors' institution, ulinastatin, as a prophylactic treatment for postoperative AE after pulmonary resection,

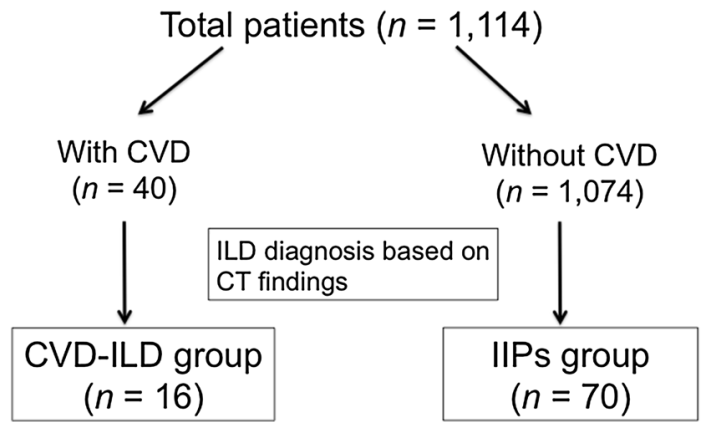

Fig. 1 Study enrolment flow chart. CT indicates computed tomography; CVD, collagen vascular disease; CVD-ILD,collagen vascular disease associated interstitial lung disease; IIPs, idiopathic interstitial pneumonias; ILD,interstitial lung disease was used for patients with simultaneous lung cancer and ILD [5]. Two days before surgery, ulinastatin was administered as a 30-min infusion of 300,000 units (U) for three consecutive days. The dose of ulinastatin for days 4 to 6 was reduced to $200,000 \mathrm{U}$, and that for days $7-9$ was further reduced to $100,000 \mathrm{U}$.

\section{Definition of postoperative event}

Postoperative complications were defined as complications that appeared within 30 days after surgery and included (1) arrhythmia requiring intervention, and (2) prolonged air leakage persisting for 7 days postoperatively and requiring pleurodesis. In addition, the 30- and 90-day mortalities were defined as death within 30 and 90 days after surgery, respectively, and included any instances of death after discharge.

Postoperative AE was diagnosed according to the following diagnostic criteria: (1) an unexplained worsening or the development of dyspnea within 30 days, (2) a new bilateral ground-glass abnormality and/or consolidation superimposed on a background reticular or honeycomb pattern on CT scan, (3) worsening hypoxemia from a known baseline arterial blood gas level, (4) the absence of infectious disease, (5) the exclusion of alternative causes including left heart failure and pulmonary embolism and (6) the identifiable cause of acute lung injury $[3,6]$. Patients with postoperative AE received steroid pulse therapy with methylprednisolone as the first line treatment.

\section{Statistical analysis}

Categorical data were presented as numbers and percentages, and continuous data were presented as the median and interquartile range (IQR). For comparing CVD-ILD and IIPs groups, the categorical data were analyzed by the Fisher's exact test or the Chi-square test, and continuous data were analyzed by the Mann-Whitney $U$ test. Overall survival (OS) was defined as the time from the operation to death from any cause, and the 5-year OS rate was estimated by the Kaplan-Meier method. Differences between the two groups were analyzed by the log-rank test. Probabilities of less than $0.05(p<0.05)$ were considered statistically significant. All statistical analyses were performed by JMP Pro 12.1 statistical software (SAS Institute, Cary, NC, USA).

\section{Ethics}

This study was approved by the Research Ethics Committee of the Tokyo Women's Medical University, Tokyo, Japan (No.3852). 


\section{Results}

Sixteen patients with CVD-ILD underwent lung resection for NSCLC at the authors' institution during the study period and their baseline characteristics are given in Table 1 . The median age was 66.5 years (IQR: 16 years), 6 patients $(37.5 \%)$ were men, and 10 patients $(62.5 \%)$ were women. There were significant differences in gender $(p=0.0201)$ and the Brinkman index $(p=0.0057)$ between the CVD-ILD and IIPs groups. ILD patterns on chest CT in CVD-ILD group were found in 10 patients with a UIP pattern, and in 6 patients with an NSIP pattern. No patients received induction chemotherapy. Pathological examinations of resected specimens showed that 7 UIP pattern cases with rheumatoid arthritis (RA) were found in the CVD-ILD group, 18 UIP pattern cases were found in the IIPs group, and one NSIP pattern was found. The median follow-up period was 34.0 months (IQR: 42.8 months). There was no significant difference in the ILD pattern on CT between the two groups. In the CVDILD group, RA was the most common CVD (Table 2). Of those, 13 patients $(81.3 \%)$ received immunosuppressive therapy consisting of prednisolone (PSL), methotrexate (MTX), tacrolimus (FK506), and mizoribine (MZ). In the immunosuppressant regimens, PSL $(n=8)$ and MTX $(n=2)$ were used as a monotherapy, and PSL with FK506 $(n=1)$, PSL with MZ, and MTX with MZ were used individually as a combination therapy.

The surgical and clinical outcomes are shown in Table 3. The most common operative procedure was lobectomy $(n=8)$, followed by segmentectomy $(n=5)$, and wedge resection $(n=3)$ in the CVD-ILD group. R0 resection was achieved in all patients except for one patient with pathological stage IV disease in the CVD-ILD group. The reinforcement of the bronchial stump in major lung resection was performed in 4 of 8 cases (50\%) in the CVD_ILD group and in 2 of 44 cases $(4.5 \%)$ in IIPs group $(p=0.0002)$, and a pedicled intercostal muscle flap was used in all cases. In the CVD-ILD group, no intraoperative complications appeared, while postoperative complications were observed in 4 patients. Postoperative $\mathrm{AE}$ developed in 1 patient (6.3\%), and BPF developed in 2 patients (12.5\%). Patients with BPF underwent the reinforcement of the bronchial stump because of the use of immunosuppressive agents. The thirty- and ninety-day mortalities were $6.3 \%$, because one patient died with postoperative AE (Table 3). Regarding the postoperative complications, no significant difference in the incidence of postoperative $\mathrm{AE}$ and a significant difference in the incidence of BPF $(p=0.0028)$ were observed between both groups. No significant differences in hospital stay, the incidence of postoperative complications, and 30- and 90-day mortalities were found between either of the groups (Table 3 ).
Table 1 Comparison of the patient characteristics

\begin{tabular}{|c|c|c|c|}
\hline Variables & CVD-ILD group & IIPs group & $p$ value* \\
\hline $\begin{array}{l}\text { Total number of } \\
\text { patients }\end{array}$ & 16 & 70 & \\
\hline Age (years) & $66.5(16)^{* *}$ & $73.0(11.3)^{* *}$ & 0.132 \\
\hline Gender & & & 0.0201 \\
\hline Male & $6(37.5 \%)$ & $62(88.6 \%)$ & \\
\hline Female & $10(62.5 \%)$ & $8(11.4 \%)$ & \\
\hline Brinkman index & $600(900)^{* *}$ & $1000(1000)^{* *}$ & 0.0057 \\
\hline $\begin{array}{l}\text { Number of comorbidi- } \\
\text { ties }\end{array}$ & & & 0.119 \\
\hline 1 & 0 & $8(11.4 \%)$ & \\
\hline 2 & $10(62.5 \%)$ & $31(44.3 \%)$ & \\
\hline 3 & $6(37.5 \%)$ & $19(27.2 \%)$ & \\
\hline $4 \leq$ & 0 & $12(17.1 \%)$ & \\
\hline ILD pattern on CT & & & 0.0709 \\
\hline UIP pattern & $10(62.5 \%)$ & $58(82.9 \%)$ & \\
\hline NSIP pattern & $6(37.5 \%)$ & $12(17.1 \%)$ & \\
\hline$\% \mathrm{VC}$ & $109.7(40.4)^{* *}$ & $108.7(34.5)^{* *}$ & 0.893 \\
\hline$\%$ DLco & $57.4(16.1)^{* *}$ & $55.5(27.6)^{* *}$ & 0.478 \\
\hline KL-6 (U/mL) & $481(342.5)^{* *}$ & $514(440)^{* *}$ & 0.699 \\
\hline $\begin{array}{l}\text { Neoadjuvant chemo- } \\
\text { therapy }\end{array}$ & 0 & $2(2.9)$ & 0.494 \\
\hline Pathology & & & 0.614 \\
\hline Adenocarcinoma & $9(56.3 \%)$ & $30(42.9 \%)$ & \\
\hline $\begin{array}{l}\text { Squamous cell carci- } \\
\text { noma }\end{array}$ & $5(31.2 \%)$ & $30(42.9 \%)$ & \\
\hline Others & $2(12.5 \%)$ & $10(14.2 \%)$ & \\
\hline Pathological stage & & & 0.153 \\
\hline I & $9(56.2 \%)$ & $40(57.1 \%)$ & \\
\hline II & $4(25 \%)$ & $11(15.7 \%)$ & \\
\hline III & $2(12.5 \%)$ & $19(27.2 \%)$ & \\
\hline IV & $1(6.3 \%)$ & 0 & \\
\hline $\begin{array}{l}\text { Median follow-up } \\
\text { (months) }\end{array}$ & $34(42.8)^{* *}$ & $30.5(35.5)^{* *}$ & 0.89 \\
\hline
\end{tabular}

* $p$ values were calculated by Fisher's exact test or the Chi-square test for categorical data, or Mann-Whitney's $U$ test for continuous data

**Continuous variables are expressed as the median and interquartile ranges which are expressed in parenthesis, and categorical variables are expressed as numbers and percentages (\%). CVD-ILD indicates collagen vascular disease-associated interstitial lung disease

IIPs idiopathic interstitial pneumonias, ILD interstitial lung disease, $C T$ computed tomography, UIP usual interstitial pneumonia, NSIP non-specific interstitial pneumonia, $V C$ vital capacity, $D L_{C O}$ carbon monoxide diffusion capacity, $K L-6$ Krebs von den Lungen- 6

The five-year OS rates were $37.5 \%$ in the CVD-ILD group and $49.2 \%$ in the IIPs group (Fig. 2), and there was no significant deference between the two groups. A total 10 deaths after surgery were observed in the CVD-ILD group during the observation period, and the causes in the CVD-ILD group were compared with those in the IIPs group (Table 4), and ILD was the second common cause 
Table 2 Types of collagen disease and the distribution of ILD patterns
Table 3 Comparison of the surgical and clinical outcomes

\begin{tabular}{lllc}
\hline Collagen Disease & ILD pattern on CT & The \\
\cline { 2 - 4 } & UIP pattern & NSIP pattern & $\begin{array}{l}\text { number of } \\
\text { cases }\end{array}$ \\
\hline Rheumatoid arthritis (RA) & 9 & 4 & 13 \\
Sjögren syndrome (SjS) & 1 & 0 & 1 \\
Dermatomyositis (DM) & 0 & 1 & 1 \\
Systemic lupus erythematosus (SLE) & 0 & 1 & 1 \\
\hline
\end{tabular}

$I L D$ interstitial lung disease, $C T$ computed tomography, UIP usual interstitial pneumonia, NSIP non-specific interstitial pneumonia

\begin{tabular}{|c|c|c|c|}
\hline Variables & CVD-ILD group $(n=16)$ & IIPs group $(n=70)$ & $p$ value* \\
\hline Operative approach & & & 0.766 \\
\hline VATS & $12(75 \%)$ & $47(67.1 \%)$ & \\
\hline Thoracotomy & $4(25 \%)$ & $23(32.9 \%)$ & \\
\hline Operative method & & & 0.418 \\
\hline Wedge resection & $3(18.7 \%)$ & $18(25.7 \%)$ & \\
\hline Segmentectomy & $5(31.3 \%)$ & $8(11.5 \%)$ & \\
\hline Lobectomy & $8(50 \%)$ & $40(57.1 \%)$ & \\
\hline Bilobectomy & 0 & $3(4.3 \%)$ & \\
\hline Pneumonectomy & 0 & $1(1.4 \%)$ & \\
\hline Operative time (min) & $163.5(115.8)^{* *}$ & $189(117.5)^{* *}$ & 0.682 \\
\hline Loss (mL) & $92.5(340.5)^{* *}$ & $145(281)^{* *}$ & 0.915 \\
\hline Intraoperative complication & 0 & $2(2.9 \%)$ & 0.494 \\
\hline Duration of drainage (days) & $4(3) * *$ & $4(3)^{* *}$ & 0.0777 \\
\hline Length of stay (days) & $11(10.5)^{* *}$ & $12(10)^{* *}$ & 0.96 \\
\hline \multicolumn{4}{|l|}{ Postoperative complication } \\
\hline Overall & $4(25 \%)$ & $19(27.1 \%)$ & 0.861 \\
\hline AE of ILD & $1(6.3 \%)$ & $6(8.6 \%)$ & 0.759 \\
\hline Prolonged air leakage & $3(18.8 \%)$ & $6(8.6 \%)$ & 0.23 \\
\hline Pneumonia & 0 & $3(4.3 \%)$ & 0.399 \\
\hline $\mathrm{BPF}$ & $2(12.5 \%)$ & 0 & 0.0028 \\
\hline Reintubation & $1(6.3 \%)$ & $2(2.9 \%)$ & 0.505 \\
\hline Tracheostomy & $1(6.3 \%)$ & $2(2.9 \%)$ & 0.505 \\
\hline Arrhythmia & $1(6.3 \%)$ & $1(1.4 \%)$ & 0.248 \\
\hline Others & 0 & $4(5.7 \%)$ & 0.328 \\
\hline 30-day mortality & $1(6.3 \%)$ & $2(2.9 \%)$ & 0.505 \\
\hline 90-day mortality & $1(6.3 \%)$ & $4(5.7 \%)$ & 0.934 \\
\hline
\end{tabular}

* $p$ values were calculated by Fisher's exact test or the Chi-square test for categorical data, or Mann-Whitney's $U$ test for continuous data

**Continuous variables are expressed as the median and interquartile ranges which are expressed in parenthesis, and the categorical variables are expressed as numbers and percentages (\%)

$C V D$ - $I L D$ indicates collagen vascular disease-associated interstitial lung disease, IIPs idiopathic interstitial pneumonias, VATS video-assisted thoracoscopic surgery, $A E$ acute exacerbation, $I L D$ interstitial lung disease, $B P F$ bronchopleural fistula of death after lung cancer in both groups. This study also compared the OS rates between CVD-ILD with a UIP pattern and IPF patients, and between RA-associated ILD (RA-ILD) and IIPs patients (Figs. 3, 4). The fiveyear OS rates were $30 \%$ in the CVD-ILD patients with a UIP pattern and $42.5 \%$ in the IPF patients, and no significant difference was found between them (Fig. 3). The five-year OS rates were $23 \%$ in the RA-ILD patients and $49.2 \%$ in the IIPs patients, and there was a significant difference between the two groups ( $p=0.0105)$ (Fig. 4). 


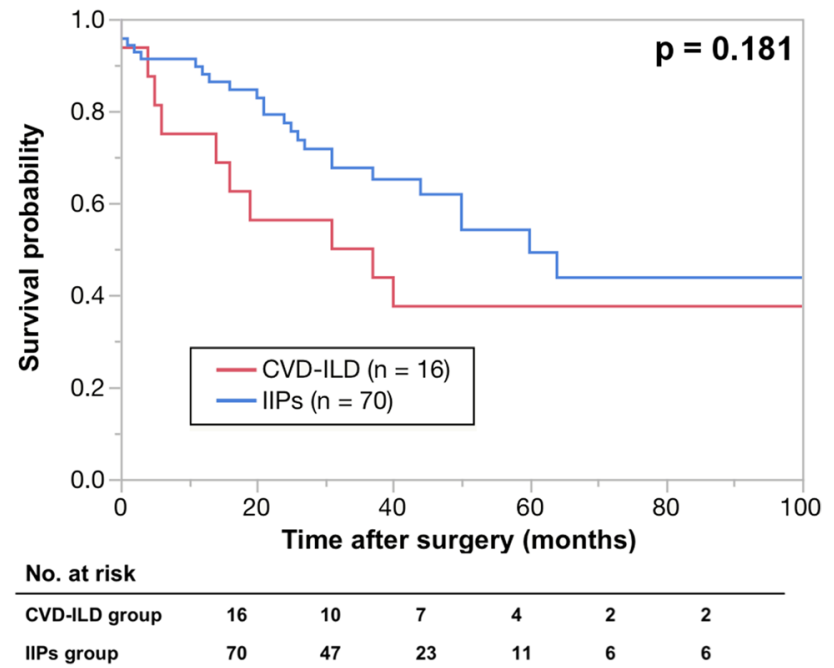

Fig. 2 Kaplan-Meier survival curves of collagen vascular diseaseassociated interstitial lung disease (CVD-ILD) andidiopathic interstitial pneumonias (IIPs) groups. The 5-year overall survival rates in patients with interstitiallung disease were $35.2 \%$ in CVD-ILD group $(\mathrm{n}=16)($ red line $)$ and $45.1 \%$ in IIPs group $(\mathrm{n}=70)$ (blue line)

Table 4 Details of the cause of death

\begin{tabular}{llll}
\hline Cause & $\begin{array}{l}\text { CVD-ILD } \\
\text { group } \\
(n=10)\end{array}$ & IIPs group $(n=26)$ & $p$ value \\
\hline Lung cancer (\%) & $5(50)$ & $16(61.5)$ & 0.769 \\
ILD (\%) & $3(30)$ & $7(27)$ & \\
Postoperative AE & 1 & 6 & \\
AE occurring $\geq$ POD 31 & 2 & 1 & \\
Others (\%) & $2(20)$ & $3(11.5)$ & \\
\hline
\end{tabular}

$C V D$ - ILD indicates collagen vascular disease-associated interstitial lung disease, IIPs idiopathic interstitial pneumonias, ILD interstitial lung disease, $A E$ acute exacerbation, $P O D$ postoperative day

\section{Discussion}

To date, the clinical features and prognosis of CVD-ILD with those of IIPs are studied and reported by several investigators. Among them, Park et al. report that (1) the common types of CVD-ILD are a UIP and non-specific interstitial pneumonia pattern according to the classification of IIPs and (2) a UIP pattern is found to more frequently coexist with RA than other CVD types [7]. Park et al. also report the (1) 3- and 5-year survival rates to be better in patients with a UIP pattern in the CVD group than those of the IIPs group, but (2) no significant difference was found between the patients with an NSIP pattern in both groups [2]. The report also suggests that IIPs may affect the prognosis of lung cancer patients more strongly than CVDILD. However, this study obtained the opposite results.

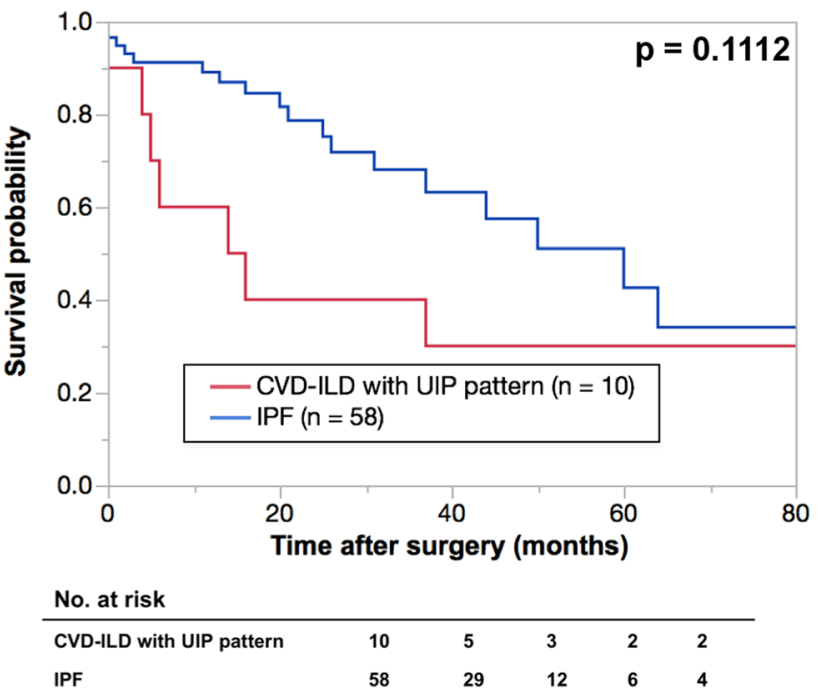

Fig. 3 Kaplan-Meier survival curves of collagen vascular diseaseassociated interstitial lung disease (CVD-ILD) withusual interstitial pneumonia (UIP) pattern and idiopathic pulmonary fibrosis (IPF) groups. The 5-year overallsurvival rates in patients with interstitial lung disease were 30\% in CVD-ILD patients with UIP pattern (n $=10)$ (red line) and $42.5 \%$ in IPF group $(n=58)$ (blue line)

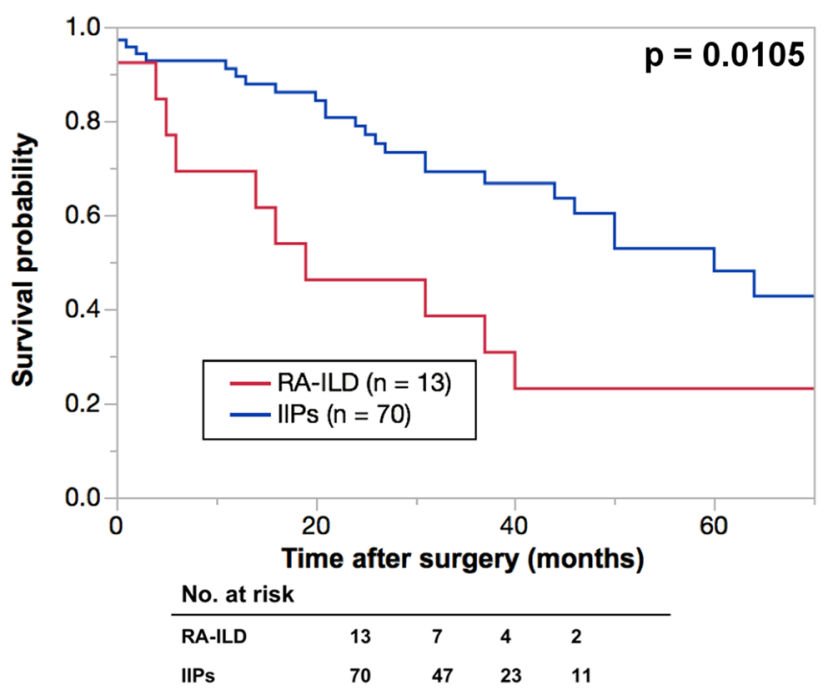

Fig. 4 Kaplan-Meier survival curves of rheumatoid arthritis-associated interstitial lung disease (RA-ILD) andidiopathic interstitial pneumonias (IIPs) groups. The 5-year overall survival rates in patients with interstitiallung disease were $23 \%$ in RA-ILD $(\mathrm{n}=10)$ (red line) and $49.2 \%$ in IIPs group $(n=70)$ (blue line)

Although no significant difference in the 5-year OS rates between CVD-ILD patients and IIPs patients was found (Fig. 2), the CVD-ILD group showed a lower OS rate. Further analysis found that (1) CVD-ILD patients with a UIP pattern showed a lower OS rate than IPF patients (Fig. 3) and (2) RA-ILD patients had a significantly lower OS rate 
than IIPs patients (Fig. 4), indicating that IIPs failed to affect the prognosis of lung cancer patients more strongly than CVD-ILD. For explaining the lower OSs in patients with CVD-ILD in this study, the following reasons were speculated. First, the state of ILDs or CVD was ignored in the survival analysis, and this might have caused some bias in selecting the adjuvant chemotherapy and the treatment of recurrence of lung cancer. Second, as Sumikawa et al. [8] and Sverzellati [9] reported, there could be a discrepancy between the diagnosis of ILDs based on the CT and pathological findings. Even in this study, CVD-ILD group included 7 patients with a UIP pattern, and the IIPs group included 18 patients with a UIP pattern. Therefore, some patients showing a UIP pattern on CT might demonstrate a UIP pattern in the pathological findings. This inconsistency might affect OS. Since the resected specimens did not always include the area of ILD, the pathological diagnoses of ILDs were thus considered to be difficult to confirm in all cases. Therefore, physicians must realize the importance of multidisciplinary discussions with pathologists in diagnosing ILDs. Another important reason to explain why the lower OSs were found in patients with CVD-ILD in this study appeared to be due to a difference in the sample size.

There are three reports regarding the 5-year survival rates in lung cancer patients with IIPs, and the 5-year survival rates in patients with NSCLC and IPF, stage IA NSCLC and IPF, and IPF and stage IA and IB NSCLC were $52 \%$ [10], 54.2\% [11], and $61.6 \%$ [12], respectively. A multicenter retrospective study with a large numbers of patients with ILD who underwent lung cancer surgery shows that the 5-year overall survival rates are $40.0 \%$ [13]. As described above, even though the diagnosis of ILD based on the CT findings might influence the results, the 5 -year survival rates in the lung cancer patients with IIPs in this study showed no major difference from the reported results, and at least, similar to IIPs, this study showed that CVD-ILD might affect the long-term results of lung cancer surgery.

Lung resection in CVD-ILD patients could lead to postoperative $\mathrm{AE}$, which is a fatal complication. However, these patients may be unable to obtain as much of a benefit as that provided by the other treatment modalities including chemotherapy and radiation therapy. Kenmotsu et al. [14] report that $9 \%$ of 104 NSCLC patients with ILD who are treated with platinum-based chemotherapy develop a chemotherapy-related exacerbation during the first-line chemotherapy. Although molecular targeting drugs, such as epidermal growth factor receptor tyrosine kinase inhibitor and anaplastic lymphoma kinase inhibitor, have recently been used to treat NSCLC, these agents can cause an exacerbation of ILD as an adverse effect. In addition, a dose reduction may be considered, because bone marrow suppression after chemotherapy in CVD-ILD patients who received immunosuppressive agents can lead to the development of severe infectious diseases. Ozawa et al. [15] report that pre-existing interstitial disease is a predictive factor of extensive radiation pneumonia. From these results, chemotherapy and radiation therapy for lung cancer with CVDILD are therefore not considered to be a more effective treatment modality than surgery. Therefore, this study considered that lung cancer patients with CVD-ILD would be candidates for surgical treatment.

The prediction and prevention of postoperative $\mathrm{AE}$ is quite important. Several reports suggest that the following items could be predictors of postoperative AE; patient age, the duration of postoperative drainage and intraoperative fluid balance [16-18], typical honeycombing and possible honeycombing patterns on the chest CT findings [19], and the $\mathrm{I} / \mathrm{M}$ ratio, which is the ratio of the peak standardized uptake value (SUV) of the interstitial lung disease area to the mean SUV of the mediastinum [20]. Although medications are used to prevent postoperative AE of ILD [5, 21], no evidence for prophylaxis has yet been established. Ulinastatin, as a prophylactic agent used in this study for postoperative $\mathrm{AE}$, showed the incidence rates of postoperative AE in this study to be $6.3 \%$ in the CVD-ILD group and $2.9 \%$ in the IIP group, and these results were more favorable than the $9.3 \%$ rate reported in a multicenter study in Japan reported by Sato et al. [22]. Recently, the effect of perioperative pirfenidone treatment for preventing postoperative AE of ILD has been reported [23-25], further investigations are expected. Hozumi et al. [26] investigate the risk factor of AE of ILD in RA patients, and a univariate analysis revealed that an older age at the time of interstitial pneumonia diagnosis, the UIP pattern on HRCT, and MTX usage are associated with AE of ILD. This study was unable to identify any predictors of AE of ILD in patients with CVD-ILD, because postoperative AE appeared in only one patient with CVD-ILD. However, Sato et al. [22] report that preoperative steroid use is an independent risk factor of postoperative $\mathrm{AE}$, and in this study, the use of immunosuppressive agents in CVD-ILD patients might be a risk factor of postoperative AE. Describing the severity of postoperative AE of ILD is also important, but it is difficult because there is no report about grading postoperative AE. Katayama et al. reported on the Japan Clinical Oncology Group postoperative complications criteria, including six thoracic complications [27]. Postoperative AE is not included in these criteria, however, grading postoperative AE may be a useful tool when thoracic surgeons make an investigation concerning postoperative AE. Future considerations should thus be expected.

Besides being an essential for treating CVD, immunosuppressive agents can cause some problems in the perioperative and long-term periods for CVD patients undergoing lung resection. First, infectious complications 
such as pneumonia caused by immunosuppression, prolonged air leakage, and BPF due to the inhibition of wound healing could be induced by immunosuppressive agents. Prolonged air leakage can cause an infection in the intrapleural space, leading to pyothorax. The temporary discontinuation of immunosuppressive agents could be acceptable as long as the state of CVDs is stable, and consultations with rheumatologists are therefore important before surgery. In this study, the CVD-ILD group showed a higher incidence of BPF than the IIPs group in spite of the reinforcement of the bronchial stump, this higher incident rate would be a risk factor for inducing serious problems, because BPF can lead to fatal pyothorax and broncho-pulmonary artery fistula (BPAF). Pyothorax developed in CVD-ILD patients treated with immunosuppressive agents and it was expected to become exacerbated. Although bronchial closure methods and a predictive score for BPF have been reported $[28,29]$, no evidence showing the effectiveness of the reinforcement of the bronchial stump to prevent BPF is available. In fact, two patients who developed BPF in the CVD-ILD group were applied with the reinforcement of the bronchial stump. In the authors' department, this procedure was routinely performed for high-risk patients including patients suffering from severe diabetes mellitus, took immunosuppressive agents, or received neoadjuvant chemoradiation therapy, because it was considered possible to avoid fatal BPAF by isolating the bronchial stump and pulmonary artery. Second, calcineurin inhibitors, such as tacrolimus could worsen the prognosis of cancer over the long term, because these agents induce tumor growth, invasion, and metastasis [30]. In this study, because only one patient took tacrolimus, a calcineurin inhibitor, this study was unable to obtain any conclusive results regarding these inhibitors, because the number of observations was insufficient. Therefore, future investigations are called to clarify their effect. Third, the doses of immunosuppressive agents can be increased upon the exacerbation of the CVD status, and bone marrow suppression due to chemotherapy may lead to a critical infection. Therefore, a decrease in the dose of chemotherapy results in an insufficient curative effect, thereby affecting the prognosis.

Because this study was a retrospective single institutional study, the number of cases investigated might be insufficient, and the small sample size could be a limitation associated with this study. The results of this study should be confirmed in a multicenter clinical study with a larger number of patients. The diagnosis of ILD in this study was based on the CT findings, because the resected specimens did not always include the area of ILD. There were a variety of CVD types and immunosuppressive treatment regimens in this study, and further investigations with a sufficient number of cases are called in the future.

\section{Conclusion}

In conclusion, this study speculated that the incidence rate of postoperative AE of ILD and mortality in CVD-ILD patients after lung cancer surgery were not higher than those of IIPs patients. CVD-ILD patients might thus have poor prognosis after lung resection for NSCLC similar to that seen in IIPs patients.

\section{Compliance with ethical standards}

Conflict of interest None declared.

Open Access This article is distributed under the terms of the Creative Commons Attribution 4.0 International License (http:// creativecommons.org/licenses/by/4.0/), which permits unrestricted use, distribution, and reproduction in any medium, provided you give appropriate credit to the original author(s) and the source, provide a link to the Creative Commons license, and indicate if changes were made.

\section{References}

1. Fukui M, Suzuki K, Matsunaga T, Oh S, Takamochi K. Outcomes of lung cancer resection for patients with combined pulmonary fibrosis and emphysema. Surg Today. 2016;46:341-7.

2. Park JH, Kim DS, Park IN, Jang SJ, Kitaichi M, Nicholson AG, et al. Prognosis of fibrotic interstitial pneumonia: idiopathic versus collagen vascular disease-related subtypes. Am J Respir Crit Care Med. 2007;175:705-11.

3. Raghu G, Collard HR, Egan JJ, Martinez FJ, Behr J, Brown KK, et al. An official ATS/ERS/JRS/ALAT statement: idiopathic pulmonary fibrosis: evidence-based guideline for diagnosis and management. Am J Respir Crit Care Med. 2011;183:788-824.

4. Travis WD, Hunninghake G, King TE Jr, Lynch DA, Colby TV, Galbin JR, et al. Idiopathic nonspecific interstitial pneumonia: report of an American Thoracic Society Project. Am J Respir Crit Care Med. 2008;177:1338-47.

5. Kanzaki M, Kikkawa T, Maeda H, Kondo M, Isaka T, Shimizu $\mathrm{T}$, et al. Acute exacerbation of idiopathic interstitial pneumonias after surgical resection of lung cancer. Interact Cardiovasc Thorac Surg. 2011;13:16-20.

6. Hyzy R, Huang S, Myers J, Flaherty K, Martinez F. Acute exacerbation of idiopathic pulmonary fibrosis. Chest. 2007;132:1652-8.

7. Kim EJ, Collard HR, King TE Jr. Rheumatoid arthritis-associated interstitial lung disease: the relevance of histopathologic and radiographic pattern. Chest. 2009;136:1397-405.

8. Sumikawa H, Johkoh T, Colby TV, Ichikado K, Suga M, Taniguchi $\mathrm{H}$, et al. Computed tomography findings in pathological usual interstitial pneumonia: relationship to survival. Am J Respir Crit Care Med. 2008;177:433-9.

9. Sverzellati N, Wells AU, Tomassetti S, Desai SR, Copley SJ, Aziz ZA, et al. Biopsy-proved idiopathic pulmonary fibrosis: spectrum of nondiagnostic thin-section CT diagnoses. Radiology. 2010;254:957-64. 
10. Voltolini L, Bongiolatti S, Bargagli E, Fossi A, Ghiribelli C, et al. Impact of interstitial lung disease on short-term and longterm survival of patients undergoing surgery for non-small-cell lung cancer: analysis of risk factors. Euro J Cardiothorac Surg. 2013;43:e17-e23.

11. Saito Y, Kawai Y, Takahashi N, Ikeya T, Murai K, Kawabata Y, et al. Survival after surgery for pathologic stage IA non-small cell lung cancer associated with idiopathic pulmonary fibrosis. Ann Thorac Surg. 2011;92:1812-7.

12. Watanabe A, Higami T, Ohori S, Koyanagi $T$, Nakashima S, Mawatari T. Is lung cancer resection indicated in patients with idiopathic pulmonary fibrosis? J Thorac Cardiovasc Surg. 2008;136:1357-63.

13. Sato T, Watanabe A, Kondo H, Kanzaki M, Okubo K, Yokoi K, et al. Long-term results and predictors of survival after surgical resection of patients with lung cancer and interstitial lung disease. J Thorac Cardiovasc Surg. 2015;149:64-70.

14. Kenmotsu H, Naito T, Mori K, Ko R, Ono A, Wakuda K, et al. Effect of platinum-based chemotherapy for non-small cell lung cancer patients with interstitial lung disease. Cancer Chemother Phramacol. 2015;75:521-6.

15. Ozawa Y, Abe T, Omae M, Matsui T, Kato M, Hasegawa H, et al. Impact of preexisting interstitial lung disease on acute, extensive radiation pneumonitis: retrospective analysis of patients with lung cancer. PloS One. 2015;10:e0140437.

16. Iyoda A, Jiang S, Amano H, Ogawa F, Matui Y, Kurouzu N, et al. Prediction of postoperative exacerbation of interstitial pneumonia in patients with lung cancer and interstitial lung disease. Exp Ther Med. 2011;2:1073-6.

17. Maniwa $T$, Isaka $M$, Nakagawa $K$, Ohde $Y$, Okumura $T$, Endo $\mathrm{M}$, et al. Chest-tube drainage is a sign of acute exacerbation of interstitial lung disease associated with lung cancer. Surg Today. 2013;43:408-11.

18. Mizuno Y, Iwata H, Shirahashi K, Takamochi K, Oh S, Suzuki $\mathrm{K}$, et al. The importance of intraoperative fluid balance for the prevention of postoperative acute exacerbation of idiopathic pulmonary fibrosis after pulmonary resection. Eur J Cardiothorac Surg. 2012;41:e161-e5.

19. Sugiura H, Takeda A, Hoshi T, Kawabata Y, Sayama K, Jinzaki $\mathrm{M}$, et al. Acute exacerbation of usual interstitial pneumonia after resection of lung cancer. Ann Thorac Surg. 2012;93:937-43.

20. Maniwa T, Endo M, Isaka M, Nakagawa K, Ohde Y, Okumura $\mathrm{T}$, et al. Acute exacerbation of interstitial lung disease with lung cancer after surgery: evaluation with
2-[18]-fluoro-2-deoxy-D-glucose positron emission tomography. Surg Today. 2014;44:494-8.

21. Ito H, Nakayama H, Yokose T, Yamaza K. Prophylaxis for acute exacerbation of interstitial pneumonia after lung resection. Asian Cardiovasc Thorac Ann. 2014;22:948-54.

22. Sato T, Teramukai S, Kondo H, Watanabe A, Ebina M, Kishi K, et al. Impact and predictors of acute exacerbation of interstitial lung diseases after pulmonary resection for lung cancer. J Thorac Cardiovasc Surg. 2014;147:1604-11.

23. Iwata T, Yoshida S, Nagato T, Nakajima T, Suzuki H, Tagawa $\mathrm{T}$, et al. Experience with perioperative pirfenidone for lung cancer surgery in patients with idiopathic pulmonary fibrosis. Surg Today. 2015. doi:10.1007/s00595-014-1071-5.

24. Iwata T, Yoshino I, Yoshida S, Ikeda N, Tsuboi M, Asato Y, et al. A phase II trial evaluating the efficacy and safety of perioperative pirfenidone for prevention of acute exacerbation of idiopathic pulmonary fibrosis in lung cancer patients undergoing pulmonary resection: West Japan Oncology Group 6711 L (PEOPLE Study). Respir Res. 2016. doi:10.1186/s12931-016-0398-4.

25. Iwata T, Yoshida S, Fujiwara T, Wada H, Nakajima T, Suzuki H, et al. Effect of perioperative pirfenidone treatment in lung cancer patients with idiopathic pulmonary fibrosis. Ann Thorac Surg. 2016. doi:10.1016/j.athoracsur.2016.05.094.

26. Hozumi H, Nakamura Y, Johkoh T, Sumikawa H, Colby TV, Kono M, et al. Acute exacerbation in rheumatoid arthritis-associated interstitial lung disease: a retrospective case control study. BMJ Open. 2013;3:e03132.

27. Katayama H, Kurokawa Y, Nakamura K, Ito H, Kanemitsu Y, Masuda N, Tsubosa Y, et al. Extended Clavien-Dindo classifyion of surgical complications: Japan Clinical Oncology Group postoperative complications criteria. Surg Today. 2016;46:668-85.

28. Uçvet A, Gursoy S, Sirzai S, Erbaycu AE, Ozturk AA, Ceylan $\mathrm{KC}$, et al. Bronchial closure methods and risk for bronchopleural fistula in pulmonary resections: how a surgeon may choose the optimum method? Interact Cardiovasc Thorac Surg. 2011:12:558-62.

29. Pforr A, Pagès PB, Baste JM, Thomas P, Falcoz PE, Barthes FL, et al. A predictive score for bronchopleural fistula established using the French database Epithor. Ann Thorac Surg. 2016;101:287-93.

30. Derynck R, Akhurst RJ. Differentiation plasticity regulated by TGF-beta family proteins in development and disease. Nat Cell Biol. 2007;9:1000-4. 